\title{
Traduction technique : les langues contrôlées au service de l'ergonomie documentaire
}

Technical Translating: How Controlled Natural Languages Can Improve

Document Ergonomics

\section{Richard Ryan}

\section{CpenEdition}

\section{Journals}

Édition électronique

URL : http://journals.openedition.org/ilcea/1090

DOI : 10.4000/ilcea.1090

ISSN : 2101-0609

Éditeur

UGA Éditions/Université Grenoble Alpes

Édition imprimée

ISBN : 978-2-84310-203-5

ISSN : 1639-6073

Référence électronique

Richard Ryan, «Traduction technique : les langues contrôlées au service de l'ergonomie

documentaire », ILCEA [En ligne], 14 | 2011, mis en ligne le 30 juin 2011, consulté le 30 avril 2019. URL http://journals.openedition.org/ilcea/1090; DOI : 10.4000/ilcea.1090

Ce document a été généré automatiquement le 30 avril 2019

(c) ILCEA 


\title{
Traduction technique : les langues contrôlées au service de l'ergonomie documentaire
}

\author{
Technical Translating: How Controlled Natural Languages Can Improve
}

Document Ergonomics

Richard Ryan

1 Dans une métaphore désormais classique, Danica Seleskovitch comparait le processus de traduction à une opération de cuisson au four au cours de laquelle certains éléments du discours étaient nécessairement transformés, pendant que d'autres, insensibles, restaient intacts (Laplace, 1994). C'est la fameuse « brioche aux raisins » : les raisins, qui résistent à la cuisson, représentent les correspondances lexicales (on parle de stabilité référentielle), tandis que la pâte qui enrobe les raisins doit être transformée pour que la brioche soit comestible. Cette transformation représente la reformulation nécessaire au passage d'une langue à une autre (ou d'un destinataire à un autre).

Dans le domaine de la traduction technique, les raisins de la brioche représentent ce que l'on appelle souvent, pour simplifier, la " terminologie », c'est-à-dire les éléments stables, monosémiques, voire, dans le meilleur des cas, biunivoques. Aux qualités gustatives de la pâte sortie du four correspond la qualité ergonomique du texte traduit : si la pâte est mal faite ou mal cuite, la brioche ne se trouvera pas en adéquation avec sa finalité première. De même pour un document technique : sa terminologie a beau être exacte, si celle-ci est mal véhiculée, le document sera rejeté par l'utilisateur.

3 Ainsi la brioche aux raisins (comprendre : la traduction) n'est acceptable que si deux critères sont satisfaits :

- les raisins sont présents (la terminologie est correcte, sans omission);

- la pâte est appétissante et savoureuse (le document est acceptable dans sa forme et dans sa fonction).

4 Face à un document technique, le traducteur se trouve de ce fait confronté à une double tâche, qui appelle une double maîtrise : s'assurer que la terminologie est conforme, mais 
aussi que le document traduit est d'une bonne qualité opérationnelle, c'est-à-dire qu'il permet à un utilisateur d'agir (recueillir des informations pertinentes, exécuter une tâche de manière efficace, prendre une décision avisée...), tout en s'intégrant dans un système documentaire cohérent.

5 En premier lieu, la conformité de la terminologie suppose de la part du traducteur un degré de maîtrise du domaine technique dont il est question, mais également des connaissances dans la discipline scientifique dénommée "terminologie», ainsi qu'en terminotique, discipline qui conjugue terminologie et informatique ${ }^{1}$. La seconde tâche $d u$ traducteur est d'assurer la qualité d'ensemble du document. C'est cette tâche qui nous intéresse ici.

6 Afin d'améliorer la qualité opérationnelle des documents techniques (univocité, sécurité, moindre coût de réalisation et de gestion...), les grands utilisateurs industriels ont développé des technolectes restreints, appelés langues contrôlées. Mais l'utilisation de celles-ci est restée largement interne aux entreprises de certains secteurs, que ce soit pour la rédaction technique, pour la traduction ou pour la pré-édition de documents destinés à la traduction automatique. Elles restent donc peu connues des traducteurs exerçant en dehors de ces secteurs, et $a$ fortiori des traducteurs indépendants.

Dans ce qui suit, nous verrons comment une connaissance des langues contrôlées, ainsi que des principes qui les sous-tendent, peut aider tout traducteur technique à améliorer la qualité de ses traductions. Dans un premier temps, nous cernerons la notion de qualité en faisant appel au concept de qualité ergonomique. Ensuite, après un bref exposé des caractéristiques des langues contrôlées, nous plaiderons pour l'intégration de certaines règles propres à celles-ci dans la bonne pratique de la traduction technique. Enfin, cette bonne pratique sera illustrée par des exemples concrets.

\section{Acceptabilité et qualité ergonomique}

8 Au plan rédactionnel, le document technique est un écrit de type informatif, surnormé et objectif, qui privilégie les fonctions phatique et métalinguistique (axe de la normalisation) et référentielle (axe de l'objectivité) [Nakbi, 2002]. Sa validation opérationnelle est subordonnée à l'effacement de son rédacteur $^{2}$ (le document est impersonnel) et à la caution de son émetteur.

L'acceptabilité d'une traduction d'un document technique suppose la conjonction de quatre critères de forme (Froeliger, 2008) :

- exhaustivité : aucune omission ;

- monosémie : un sens et un seul ;

- précision : maîtrise de l'interprétation;

- accessibilité : style standard conforme aux bonnes pratiques.

10 L'exhaustivité et la monosémie sont dans les raisins de la brioche; la précision et l'accessibilité sont dans la pâte. Conscients que ces critères ne sont pas étanches et qu'ils peuvent se recouper, nous posons que les deux premiers critères relèvent de la maitrise du contenu informationnel du document, c'est-à-dire ce que l'on pourrait appeler les données du texte, son contenu « expert », dont le traducteur doit assurer l'exactitude : le traducteur reporte. En même temps, les deux derniers critères délimitent un champ d'action où le traducteur se trouve face au destinataire du document traduit, et à la nécessité de se faire comprendre de lui : le traducteur rédige. Pour répondre à ces deux 
derniers critères, à la différence des deux premiers, le traducteur dispose d'une importante marge de manœuvre, car il lui est permis (voire conseillé, et même enjoint) d'améliorer la qualité fonctionnelle du document source, souvent défaillante, lui ajoutant ainsi de la valeur. Le traducteur a toujours un « devoir d'améliorer » (Ryan, 2009). C'est dans ces deux derniers critères que réside ce que nous appelons ici la "qualité ergonomique » du document.

11 Nous entendons par "qualité ergonomique» un ensemble d'aspects formels d'un document qui contribuent à son acceptabilité auprès des utilisateurs auxquels le document est destiné. Nous nous référons au concept d'ergonomie ${ }^{3}$ dans son acception anglo-saxonne, centrée sur les facteurs humains, et plus spécialement à l'ergonomie cognitive. Cette branche de l'ergonomie s'intéresse aux systèmes, le plus souvent informationnels, qui imposent une charge mentale à l'utilisateur (décryptage, analyse, mémoire de travail, intégration de schémas mentaux...). Le document technique fait manifestement partie de ces systèmes.

12 La qualité ergonomique d'un document est la résultante de plusieurs variables, principalement son "utilisabilité», qui donne la mesure de ses performances opérationnelles, et sa crédibilité, ou le crédit dont il jouit auprès de ses utilisateurs.

\section{L'utilisateur}

Qui utilise le document technique? Nous pouvons distinguer différents utilisateurs: «l'utilisateur de terrain», c'est-à-dire la personne chargée d'agir à la lecture du document (par exemple, un technicien de maintenance), mais aussi d'autres utilisateurs situés à un plus haut niveau d'expertise, tel que «l'utilisateur responsable » dont le rôle est de contrôler l'utilisation du document et de veiller au respect d'un standard documentaire, ou bien « l'utilisateur décisionnel » qui définit ce standard pour répondre à des contraintes économiques ou réglementaires. Ainsi, le chercheur scientifique qui fait traduire un article qu'il a lui-même rédigé s'attendra à ce que le traducteur tienne compte des normes de présentation de la revue dans laquelle l'article paraîtra, et dont l'équipe éditoriale fixe un standard en fonction des usages de l'édition scientifique. Une entreprise peut choisir une politique documentaire tenant compte non seulement des performances pratiques d'un document, mais également de sa gestion, de sa traçabilité, de son coût, de sa mise à jour, de sa conformité à une norme, ou encore de son utilisation par divers acteurs opérant à des niveaux différents dans des contextes multiples. Enfin, rappelons que l'utilisateur peut-être un non-natif: c'est souvent le cas pour l'anglais, mais aussi pour le français dans les pays de la francophonie.

Ainsi l'acceptabilité d'un document technique, si elle intègre le jugement de l'utilisateur de terrain, dépend aussi de l'appréciation d'autres acteurs. La cible du document technique est donc en réalité un ensemble d'utilisateurs caractérisés par des attentes diverses, mais convergentes. Dans ce qui suit, le terme « utilisateur » sous-entendra cette pluralité.

\section{L'utilisabilité}

La qualité ergonomique d'un document se reflète dans son "utilisabilité », terme calqué sur l'anglais usability et défini dans la norme internationale ISO 9241 («Ergonomie de 
l'interaction homme-système », partie 110, 2006) : «Degré selon lequel un produit peut être utilisé par des utilisateurs identifiés, pour atteindre des buts définis avec efficacité, efficience et satisfaction dans un contexte d'utilisation spécifié. » L'efficacité d'un produit (ici, un document technique traduit) se mesure à sa capacité à permettre à l'utilisateur d'atteindre un but; son efficience traduit le gain d'effort ou de temps nécessaire à l'utilisateur pour atteindre ce but, deux variables à minimiser. L'efficacité et l'efficience du produit concourent à ce que nous appelons ici l'utilitét du produit, dans son acception économique. Quant à la satisfaction de l'utilisateur, celle-ci dépendra de la facilité d'appropriation, du confort d'interaction et de la robustesse (ou fiabilité opérationnelle) du produit, tels que l'utilisateur les perçoit. C'est ce que nous appelons ici la commodité ou la praticité ${ }^{6}$ du produit. Globalement, l'utilisabilité d'un document, ou plus simplement son adéquation ${ }^{7}$, relève du traitement graphique de l'information qu'il contient, et de sa structuration selon une logique d'utilisation (et non de conception, par exemple).

\section{La crédibilité}

16 À la notion subjective de satisfaction, nous en ajouterons ici une autre, suffisamment importante pour mériter une place à part, à savoir la crédibilité du document, mesure de la confiance qu'il inspire, ou plutôt qu'inspire son auteur ou émetteur (ou son traducteur). L'importance de la maîtrise du contenu technique d'un texte à traduire pour assurer la crédibilité du texte cible et ne pas nuire à l'émetteur du texte source est reconnue (par exemple, dans le domaine économique, Percebois, 2004). Mais au-delà de cette maîtrise du contenu expert, la crédibilité d'un document technique est tributaire de l'autorité qu'il possède par la légitimation que lui apporte son émetteur (autorisation, qualification, approbation, validation...) et de ce que nous appelons ici sa justesse $e^{8}$, caractère apprécié par la pertinence du document (à-propos, opportunité...) et par sa fiabilité (véracité, cohérence, sincérité...), telles que ces qualités sont perçues et évaluées par l'utilisateur'. Par ailleurs, la notion de " convention textuelle» a été invoquée pour rendre compte de la reconnaissance par l'utilisateur de ce caractère de justesse: les conventions textuelles sont à la fois des «marqueurs de différenciation», des " déclencheurs d'attentes déterminées chez le récepteur », et des " balises sur le parcours herméneutique » (Reiss, 1995). Le traducteur doit veiller à conserver ou, le cas échéant, à créer ces caractéristiques de justesse dans le document cible. Rappelons enfin que la crédibilité des documents en général est menacée par la prolifération de sources documentaires "sauvages» accessibles par les réseaux électroniques (Rheault, 1999): l'authenticité même d'un document technique peut ainsi être contestée. C'est par la normalisation que nous conférons aux documents non seulement une homogénéité fonctionnelle, mais aussi une autorité, en ce que la norme constitue elle-même un critère d'authenticité. D'où la grande importance de respecter les normes et les usages de présentation ${ }^{10}$, en même temps que la conformité terminologique. Le schéma 1 reprend, en les articulant, ces différentes notions. 
Schéma 1. - Analyse de la qualité ergonomique d'un document.

\begin{tabular}{|c|c|c|c|c|c|c|}
\hline \multicolumn{7}{|c|}{ Qualité ergonomique : précision et accessibilité } \\
\hline \multicolumn{3}{|c|}{ « Utilisabilité » ou adéquation } & \multicolumn{4}{|c|}{ Crédibilité } \\
\hline \multicolumn{2}{|c|}{ Utilité } & \multirow{3}{*}{$\begin{array}{c}\text { Commodité, } \\
\text { praticité } \\
\text { Satisfaction } \\
\text { Le document est } \\
\text { facile et } \\
\text { agréable à } \\
\text { utiliser }\end{array}$} & \multicolumn{2}{|c|}{ Autorité } & \multicolumn{2}{|c|}{ Justesse } \\
\hline Efficacité & Efficience & & Légitimité & Authenticité & Pertinence & Fiabilité \\
\hline $\begin{array}{l}\text { J'atteins } \\
\text { mon but } \\
\text { grâce au } \\
\text { document }\end{array}$ & $\begin{array}{c}\text { Je fais des } \\
\text { économies } \\
\text { grâce au } \\
\text { document }\end{array}$ & & $\begin{array}{l}\text { Le document } \\
\text { est approuvé }\end{array}$ & $\begin{array}{l}\text { Le document } \\
\text { n'est pas un } \\
\text { faux }\end{array}$ & $\begin{array}{c}\text { C'est le } \\
\text { bon } \\
\text { document }\end{array}$ & $\begin{array}{l}\text { Je peux } \\
\text { me fier au } \\
\text { document }\end{array}$ \\
\hline \multicolumn{3}{|c|}{$\begin{array}{l}\text { Conception, traitement graphique de } \\
\text { l'information }\end{array}$} & \multicolumn{4}{|c|}{$\begin{array}{l}\text { Normalisation, conventions textuelles, conformité du } \\
\text { style }\end{array}$} \\
\hline
\end{tabular}

17 Notre définition de la crédibilité d'une traduction diffère de celle admise dans la traduction littéraire, qui requiert l'effacement du traducteur, de manière à «faire croire au lecteur-consommateur qu'il a affaire directement, contiguement presque, à l'auteurproducteur de l'œuvre, et non pas à un intermédiaire » (Nakbi, 2002, p. 24). La crédibilité d'un document technique suppose l'effacement de l'auteur au profit du seul contenu et de la valeur opérationnelle de celui-ci, dont le traducteur est garant.

\section{Un risque du métier}

18 Un document, aussi adéquat soit-il en réalité, sera inopérant dès lors que l'utilisateur peut mettre en doute son autorité, sa pertinence ou sa fiabilité, c'est-à-dire si le document ne lui parait pas crédible. Or, un document traduit est susceptible d'avoir été mal traduit, à plus forte raison un document technique, le traducteur n'étant pas forcément un spécialiste du domaine traité. De plus, un préjugé défavorable pénalise souvent les traducteurs techniques, soupçonnés (à tort ou à raison) d'être des littéraires de cœur, formés à la belle page. C'est ainsi qu'un doute sur la qualité d'une traduction technique peut susciter un refus, un style décalé ou une présentation inhabituelle étant tout aussi rédhibitoires qu'une grossière erreur de terminologie. On peut anéantir la crédibilité d'un document par des erreurs de registre ou tout simplement par un mauvais choix de police de caractères.

19 Si bons que soient les raisins, la brioche ne vaut rien si la pâte est mauvaise. Mais une pâte même bonne peut nous paraître suspecte. C'est ainsi qu'un document qui n'inspire pas confiance sera écarté par précaution. Un développement du modèle de la brioche aux raisins est proposé au schéma 2 : la partie grisée est celle qui nous concerne ici. 
Schéma 2. - Le modèle de la brioche aux raisins.

\begin{tabular}{|c|c|c|c|c|c|}
\hline $\begin{array}{l}\text { Constituants } \\
\text { de la brioche } \\
\text { (Selesko- } \\
\text { vitch) }\end{array}$ & Nature & $\begin{array}{l}\text { Critères de } \\
\text { forme } \\
\text { (Froeliger) }\end{array}$ & $\begin{array}{l}\text { Actions du } \\
\text { traducteur }\end{array}$ & Outils & Qualités visées \\
\hline Raisins & $\begin{array}{l}\text { Contenu } \\
\text { expert }\end{array}$ & $\begin{array}{l}\text { Exhaustivité } \\
\text { et } \\
\text { monosémie }\end{array}$ & $\begin{array}{l}\text { Report des } \\
\text { correspondances }\end{array}$ & $\begin{array}{l}\text { Bases } \\
\text { terminologiques, } \\
\text { documentation en } \\
\text { langue cible }\end{array}$ & $\begin{array}{l}\text { Conformité } \\
\text { terminologique, } \\
\text { exactitude, } \\
\text { intégralité }\end{array}$ \\
\hline Pâte & $\begin{array}{l}\text { Facteur } \\
\text { humain }\end{array}$ & $\begin{array}{l}\text { Précision et } \\
\text { accessibilité }\end{array}$ & $\begin{array}{l}\text { Reformulation, } \\
\text { contrôle } \\
\text { ergonomique, } \\
\text { amélioration }\end{array}$ & $\begin{array}{l}\text { Bonnes pratiques } \\
\text { de rédaction, } \\
\text { langues } \\
\text { contrôlées }\end{array}$ & $\begin{array}{l}\text { "Utilisabilité », } \\
\text { crédibilité : } \\
\text { qualité } \\
\text { ergonomique }\end{array}$ \\
\hline
\end{tabular}

\section{Les langues contrôlées : une aide à la rédaction au service du destinataire}

"d'expert à expert ${ }^{11}$ ». La communication technique grand public, qui fonctionne d'expert à consommateur (vulgarisation, notices, conseils, modes d'emploi, manuels de dépannage...), fait intervenir d'autres facteurs, dont la motivation de l'utilisateur ou l'image de marque de l'émetteur. Si tout document technique doit convaincre et rassurer l'utilisateur, un document destiné au grand public doit aussi le séduire, ce qui n'est pas du ressort des langues contrôlées. Nous présupposons donc que notre utilisateur est motivé d'emblée, et que la finalité du document traduit est de lui faire acquérir des connaissances, exécuter des tâches, ou prendre des décisions sous contrainte de rentabilité économique.

Une langue contrôlée (LC) est un sous-ensemble d'une langue naturelle contrainte par des règles restrictives. Celles-ci peuvent être lexicales, syntaxiques, discursives, textuelles ou pragmatiques. Le but historique de l'utilisation des LC dans l'industrie, qui remonte aux années 1970-1980, était de réduire l'ambiguïté et la complexité des documents techniques afin de diminuer les coûts directs liés à l'élaboration, à la gestion et à la traduction de la documentation, ainsi que les coûts indirects des «ratés» de la communication. Ces « ratés » pouvaient provenir soit d'erreurs de lecture, soit d'une mauvaise interprétation d'un texte, conduisant au moins à une perte de temps ou d'énergie, au pire au gaspillage, à la malfaçon ou à l'accident. Il s'agissait en somme d'améliorer ce qu'on appelait alors la "lisibilité » des documents, ceci par une double démarche de simplification et de normalisation (Ryan, 2008) : l'ergonomie documentaire était encore à ses débuts ${ }^{12}$.

Précisons que par définition, les LC respectent les règles constitutives de la langue naturelle dont ils constituent des sous-ensembles. Ainsi les différentes LC appartenant à une même langue naturelle sont miscibles en toutes proportions entre elles et avec la langue générale. De ce fait, le rédacteur peut appliquer, à son gré et au besoin, telle ou telle règle de telle ou telle $\mathrm{LC}$, et non d'autres, et cela systématiquement ou ponctuellement. Les règles des LC constituent ainsi un ensemble d'outils auxquels le traducteur technique peut faire appel à discrétion (Ryan, 2009).

L'industrie utilise actuellement un très grand nombre de LC, surtout des sous-ensembles de l'anglais, les plus anciennes d'entre elles depuis une trentaine d'années ${ }^{13}$. Deux 
grandes LC se sont imposées au fil des ans en tant que références quasi canoniques: Aerospace and Defense Simplified Technical English ou ASD-STE 100 (1986) ${ }^{14}$ (appelé souvent « anglais simplifié »), et Attempto Controlled English ou ACE (1999) [Fuchs et al., 1999] ${ }^{15}$. La première s'est développée d'une manière empirique pour la rédaction de documents de maintenance (descriptions, mises en garde, instructions), d'abord dans l'industrie aéronautique. La seconde est conçue comme une LC non ambiguë, apte au traitement informatique, pour la rédaction de spécifications (par exemple, de logiciels). Il existe une version française d'ASD-STE 100, dénommée «français rationalisé », publiée en 1990 par le Groupement des Industries françaises aéronautiques et spatiales (GIFAS) [Barthes, 1999].

Un recensement des règles de rédaction reprises dans un ensemble de LC a trouvé quelque 70 règles, dont 42 réputées essentielles pour la bonne lisibilité des textes techniques (Reuther, 2003). Une étude portant sur huit sous-ensembles d'anglais contrôlé (O'Brien, 2003) a dégagé une liste de 22 règles de rédaction susceptibles de rendre des textes en anglais plus faciles à traduire vers d'autres langues, en les rendant plus simples et moins ambigus. Dans le même but, Muegge (2007) a développé CLOUT ${ }^{\mathrm{TM}}$ (Controlled Language Optimized for Uniform Translation). Même si ces différentes règles sont d'abord conçues pour améliorer la «traductibilité » des textes sources en anglais vers d'autres langues dans une démarche de localisation, le but est toujours de produire un texte simple, maniable, homogène et univoque, facile à mettre à jour et à gérer.

Ajoutons que les LC sont souvent associées à des préconisations macrotypographiques (listes, tableaux, rubrication, mise en page...). Rappelons à ce titre que le traducteur technique doit connaître :

- les grandes lignes de la norme ISO 31, 1992 (ISO 80 000, 2009) «Grandeurs et unités » (typographie des notations scientifiques);

- le système international des unités et les règles typographiques afférentes ;

- les règles d'emploi de l'espace insécable, du trait d'union, du tiret et du signe " moins »;

- le maniement d'un éditeur d'équations.

Des ouvrages de référence portant sur les bonnes pratiques d'édition scientifique et mathématique internationale sont ici indispensables; par exemple, Council of Science Editors (2006), Swanson (2000). La macrotypographie est traitée par Bringhurst (2004).

La structuration de certains documents en LC (par exemple, les manuels de maintenance) est également contrainte par des règles textuelles visant une meilleure utilisabilité.

Si certaines règles des LC sont probablement universelles (biunivocité, chronologie, balisage et rubrication...), d'autres sont étroitement liées à leur langue source (le plus souvent l'anglais) et ne sont pas toujours transposables à d'autres langues. Les bonnes pratiques de rédaction en cours dans les pays anglophones ont été souvent importées sans tenir compte des différences de langue (par exemple, employer la voix active de préférence à la voix passive, préférer les mots courts, mettre le sens dans le verbe). Il n'est pas sûr que ces recommandations soient toujours pertinentes en français (du moins en français métropolitain...). L'applicabilité d'autres consignes est sujette à caution (longueur des segments, modalité, présuppositions...). Cependant, on peut constater une certaine uniformisation des pratiques sous l'effet de la mondialisation et de l'omniprésence de l'anglais dans le domaine technique. 


\section{Règles des langues contrôlées applicables dans le domaine de la traduction technique} d'améliorer ainsi son acceptabilité. L'application judicieuse des LC peut à la fois majorer l'utilisabilité du document en augmentant sa lisibilité et sa maniabilité (sa commodité), et renforcer sa crédibilité en le normalisant. De plus, la mise en œuvre des LC présuppose un effort d'analyse pragmatique $d u$ texte source, qui a pour effet de forcer un rapprochement du traducteur et de l'utilisateur, favorisant une orientation plus résolument «cibliste" (Ladmiral, 1986). Le souci du traducteur d'alléger la tâche de l'utilisateur ne manque pas de transparaître dans le texte cible, contribuant encore à la crédibilité du document.

Il nous semble que les règles suivantes sont susceptibles de trouver une application très large dans notre domaine, puisque les finalités d'un document technique sont, dans une grande mesure, universelles. Certaines d'entre elles sont déjà validées par une pratique existante, ou bien préconisées dans des manuels de style, ou même intégrées dans des spécifications industrielles ${ }^{16}$. Leur choix repose également pour une grande part sur l'expérience professionnelle du présent auteur au cours d'une longue pratique de la traduction scientifique et technique. La miscibilité des LC nous permet de considérer ces règles comme des recommandations de bonne pratique à suivre au fil des besoins: en somme, comme une panoplie d'outils dans laquelle nous pouvons puiser à discrétion.

Chaque règle est illustrée par un ou plusieurs exemples commentés de traductions à partir de l'anglais. Nous ne prétendons pas démontrer de manière incontestable la supériorité opérationnelle de chaque proposition à partir d'une instance isolée, sans contexte ni cotexte, et dont le choix est orienté. Il s'agit seulement de présenter des exemples concrets d'application des règles.

\section{Règles lexicales}

\section{Règle 1}

Utiliser un vocabulaire général restreint, volontairement terne et répétitif.

\section{Exemple}

Choisir « mais » au lieu de « cependant », « toutefois », « en revanche »...; «faire " pour " effectuer ", " réaliser », « exécuter ", " procéder à ", « opérer », « accomplir »...; «si » pour "à condition de/que ", " pourvu que », « au cas où ", « en cas de "; « près de " pour « à proximité de », « et " pour « ainsi que », etc.).

Cette économie lexicale a pour effet de réduire l'expressivité du texte, qualité accessoire dans un document technique.

\section{Règle 2}

Réduire ou éviter la synonymie - no elegant variation ${ }^{17}$. Conserver l'homogénéité lexicale, ce qu'on appelle en anglais : consistency.

\section{Exemple}

Dans un article de recherche :

This work focuses on the nutritional value of sainfoin. Herba medica offers numerous advantages, including [...]. 
*18 «Ce travail porte sur la valeur nutritive du sainfoin. En effet, l'herba medica présente de nombreux avantages, dont [...] $»$ devient :

«Ce travail porte sur la valeur nutritive du sainfoin, plante qui présente de nombreux avantages, dont [...]. »

Herba medica est un synonyme de sainfoin, mais son emploi ici pouvait laisser supposer que l'auteur voulait parler d'autre chose.

\section{Règle 3}

Définir les sigles et acronymes à la première occurrence. Annoncer un sigle pour remplacer une désignation qui va réapparaître souvent par la suite.

Exemple

Dans un article de recherche :

To analyse the oil we opted for gas phase chromatography / mass spectrometry. [...] The results of the gas phase chromatography / mass spectrometry are given in Table 1.

«Pour analyser l'huile, nous avons choisi la chromatographie en phase gazeuse couplée à la spectroscopie de masse (CPG-SM). [...] Le tableau 1 présente les résultats de la CPG-SM.»

Notons que le sens, équivoque, de la barre oblique (slash) est explicité, et que la voix active dans la deuxième phrase ajoute à sa concision.

\section{Règles syntaxiques}

\section{Règle 1}

Éviter l'ellipse. Rétablir les omissions.

Exemple

Dans un mode opératoire industriel :

Take the part out of the oven, let it cool down and do a check.

* «Retirer la pièce du four, laisser refroidir et contrôler » devient :

« Retirer la pièce du four, la laisser refroidir puis vérifier sa conformité. »

En effet, « do a check » est équivoque (contrôler quoi ? à quelle fin ?). Renseignement pris, il s'agit de s'assurer que l'aspect de la pièce est conforme. Par ailleurs, «and » ici signifie " puis".

\section{Règle 2}

Utiliser les pronoms personnels "nous» (le fabricant) ou "vous" (l'utilisateur) pour remplacer des tournures passives ou impersonnelles. Il est vrai que celles-ci sont encore usuelles en français (même si le français rationalisé GIFAS préconise " nous » et " vous »). L'usage en anglais évolue vers les tournures personnelles (préconisées dans la plupart des anglais contrôlés, notamment ASD-STE 100), qui permettent d'éviter la voix passive, moins explicite que la voix active puisque l'agent n'est pas nommé. Cette préférence gagne du terrain en français.

\section{Exemples}

Dans un document technico-commercial :

Spares will be supplied while stocks last.

* «Les pièces seront fournies jusqu'à épuisement des stocks » (par qui ?) devient :

«Nous fournirons les pièces jusqu'à épuisement des stocks.» (Le fournisseur

s'engage.)

Dans un mode opératoire industriel :

Make sure you wear gloves [when handling materials].

* « Il faut porter des gants » (information) devient :

«Vous devez porter des gants » (exhortation ciblée) 
ou même :

«Porter des gants» (injonction).

Les utilisateurs sont mieux protégés par une formulation directive.

\section{Règle 3}

Reformuler pour limiter la subordination.

Exemple

Dans une notice, une liste de tâches à exécuter :

The pressure gauge should be read every month.

* «La pression, dont la valeur doit être relevée tous les mois, se lit sur la jauge. » Cette traduction précise, au moyen d'une subordination, que c'est la valeur de la pression qui est relevée, information évidente pour un utilisateur de terrain, mais non explicitée. Sans la subordination, nous aurons :

"La valeur de la pression doit être relevée tous les mois. Elle se lit sur la jauge. » Mais on peut aller plus loin : "should» est équivoque (le should des notices indique une nécessité technique dans les conditions normales, mais laisse une marge d'appréciation). La forme pronominale efface l'agent (qui lit la jauge?). Nous aurons :

"Vous relevez tous les mois la valeur de la pression à la jauge. »

Un utilisateur de terrain peut, certes, préférer la première traduction, plus complice et moins directive. Mais la seconde présente l'avantage technique de ne laisser aucune marge d'interprétation, restriction qui protège l'utilisateur.

\section{Règle 4}

Éliminer les marqueurs de modalité lorsque l'évaluation subjective qu'ils sollicitent est parasitaire. Il s'agit souvent d'une intrusion de l'énonciateur qui ajoute son propre commentaire sur la nécessité, probabilité, etc., d'une action ou d'un état, envisagée d'un point de vue autre que celui de l'utilisateur.

\section{Exemples}

Dans une spécification :

A user should be able to adjust the distance manually.

* « Un utilisateur doit pouvoir régler la distance manuellement... » devient :

«L'utilisateur règle la distance manuellement... »

L'auteur projette le souci du concepteur ou du commanditaire qui souhaite que l'utilisateur puisse régler la distance manuellement. Mais ce souci n'a pas à être explicité dans une spécification, qui n'est qu'une pure description.

Plus loin dans la même spécification, l'auteur projette un souci de contenter l'utilisateur: If the user wants to adjust the distance they can do it manually.

* «Si l'utilisateur désire régler la distance, il peut le faire manuellement...» devient également :

«L'utilisateur règle la distance manuellement... »

Dans un article de recherche :

Using this device we were able to check...

* «Grâce à cet appareil, nous avons pu vérifier... » devient :

«À l'aide de cet appareil, nous avons vérifié... »

ou

«Cet appareil nous a permis de vérifier... »

La réussite de l'action ne fait aucun doute, ou alors il s'agissait de préciser que c'est seulement grâce à cet appareil que nous l'avons réussi.

Dans un mode opératoire industriel :

The distance must not be greater than $10 \mathrm{~cm}$. 
* « La distance ne doit pas excéder $10 \mathrm{~cm} »$ devient :

«La distance maximale autorisée est de $10 \mathrm{~cm}$.»

Ici «must» marque le raisonnement de l'émetteur du document (il y a un risque), qui n'est pas celui de l'utilisateur (il y a une règle).

41 À noter que l'obligation contractuelle peut être exprimée par le présent de l'indicatif à valeur prescriptive ${ }^{19}$ (équivalent ici à shall en anglais) ${ }^{20}$.

\section{Exemples}

Dans un contrat :

The manufacturer shall ensure quality control checks are carried out on raw materials.

* «Le fabricant devra assurer le contrôle des matières premières » devient :

«Le fabricant assure le contrôle des matières premières. "

Dans un contrat :

The supplier undertakes to warn the buyer if a delivery is delayed.

* «Le fournisseur s'engage à prévenir l'acheteur dans l'éventualité d'un retard de livraison » devient :

«Le fournisseur prévient l'acheteur de tout retard de livraison. »

Dans un contrat :

The contractor shall be responsible for making sure health and safety regulations are obeyed.

* «Il incombe au contractant de s'assurer que les règles d'hygiène et de sécurité sont respectées » devient :

"Le contractant assure le respect des règles d'hygiène et de sécurité. »

Exprimer l'obligation par l'indicatif, en la présentant comme réalisée et générale, favorise le respect de la disposition elle-même. Ces exemples relèvent également de l'économie lexicale préconisée plus haut.

\section{Règles textuelles}

\section{Règle 1}

Veiller à la concision du texte. Le coefficient de foisonnement appliqué aux traductions vers le français, notamment à partir de l'anglais, rend compte, certes, d'une réalité statistique. Mais ce foisonnement n'est pas fatal et résulte parfois de la sur-traduction. Le français peut être plus concis que l'anglais, comme le montrent certains de nos exemples.

Éviter les tournures telles que «Il est intéressant de constater que... » pour traduire " Interestingly, (préférer : « Notons que...»), « Il faut garder à l'esprit que... » pour «We must/should bear in mind that... » (préférer : « Rappelons que... »).

\section{Règle 2}

Utiliser des segments courts, mais pas trop courts : les phrases doivent compter entre 4 et 25 mots ; les paragraphes comptent plus d'une phrase, mais pas plus de sept.

Ces dernières consignes, inspirées des recherches sur l'empan de lecture, sont souvent énoncées dans les recommandations pour la rédaction en anglais, même en dehors du domaine technique. Il n'est pas certain qu'elles soient toujours transposables en français, où la segmentation du discours met en valeur le membre de phrase. Il est alors possible de construire des phrases longues qui, bien ponctuées, sont parfaitement limpides, ce qui semble plus difficile en anglais. La notion de segment dans ce cas est certainement à moduler selon la langue. 


\section{Règle 3}

L'ordre textuel reflète l'ordre temporel (l'outil précède l'action, la cause précède l'effet).

\section{Exemples}

Dans un mode opératoire :

Before removing the board make sure the power is switched off.

* «Avant de retirer la carte, s'assurer que l'alimentation est coupée » devient :

«S'assurer que l'alimentation est coupée puis retirer la carte »

ou même

«1. S'assurer que l'alimentation est coupée.

2. Retirer la carte. »

Dans un mode opératoire :

Remove the board with pliers.

* « Retirer la carte à l'aide d'une pince » devient :

«Utiliser une pince pour retirer la carte.»

Dans un descriptif technique :

The flap is actuated by the rod, which is actuated by the pedal.

* « Le volet est actionné par la tringle, elle-même actionnée par la pédale » devient :

«La pédale actionne la tringle, qui actionne le volet. »

Notons que dans ce dernier cas la voix active remplace la voix passive, comme le préconisent ASD-STE 100 et le français rationalisé GIFAS.

On objectera que dans ces exemples, changer l'ordre textuel entraîne une inversion thème-rhème qui fausse l'équivalence de l'énoncé. Mais les effets de cette modification, délétères ou bénéfiques, dépendront du but communicatif : différents arguments peuvent être avancés dans l'un ou l'autre sens, selon le cas (protection, motivation, économie, lisibilité, cohérence, normalisation...). Il est vrai que les langues contrôlées privilégient la fonction assertive du langage et n'abordent pas directement la question de la focalisation.

\section{Règle 4}

Séparer les différentes fonctions discursives (descriptions, instructions, mises en garde...) et leurs formes correspondantes (en français : indicatif et infinitif à valeur d'impératif, respectivement). Ordonner les différentes fonctions selon une séquence opérationnelle correspondant à un parcours cognitif : montrer, expliquer, mettre en garde, préparer, faire exécuter (ou du point de vue de l'utilisateur : voir, appréhender, se prémunir, se préparer, exécuter).

Exemple

Dans une notice :

The screen you can use to monitor the process should be cleaned regularly. Wipe with a damp cloth. Repeat at least once a week. Do NOT use organic solvents.

* «L'écran qui permet de suivre le procédé doit être nettoyé régulièrement. Essuyer avec un tissu mouillé préalablement à l'eau. Répéter au moins une fois par semaine. Attention : ne jamais utiliser des solvants organiques » devient :

«Un écran vous permet de suivre le procédé. Vous devez nettoyer cet écran au moins une fois par semaine.

Attention : ne jamais utiliser de solvant organique.

1. Mouiller un tissu à l'eau.

2. Utiliser ce tissu pour essuyer l'écran. »

La mise en garde précède les instructions (c'est plus prudent). Celles-ci sont présentées dans un ordre chronologique marqué par la numérotation.

\section{Règle 5}

Utiliser des listes : une liste à tirets ${ }^{21}$ lorsque l'ordre n'importe pas (par exemple, une liste 
d'outils), une liste numérotée quand l'ordre importe (séquence, rang) ou quand la liste est longue ou encore quand on se réfère dans le texte aux items de la liste.

\section{Exemples}

Dans un article de recherche :

Our purpose is both to assess the variability in essential oils from different parts of the plant and to look at the variability in oils from different plants.

* «Nous nous proposons d'évaluer la variabilité des huiles essentielles des différentes parties de la plante, d'une part, et la variabilité des huiles issues de différentes plantes, d'autre part » devient :

« Nous nous proposons d'évaluer la variabilité des huiles essentielles:

- issues des différentes parties de la plante ;

- issues de différentes plantes. »

Dans un rapport technico-commercial :

One of the first issues was meeting local market needs; the second step is to address the international market.

* «Un des premiers enjeux était de répondre aux besoins du marché local. Dans un second temps, l'objectif est de s'implanter sur le marché international » devient :

« Nos deux objectifs :

1. Répondre aux besoins du marché local ;

2. S'implanter sur le marché international. »

\section{Règle 6}

Dans une séquence numérotée d'instructions, donner une seule instruction par item. Écrire le résultat de l'action à sa suite.

Exemple

Dans une notice :

When you press key A a window opens. Now click B to run the application.

«Quand vous appuyez sur la touche A une fenêtre s'ouvre, puis vous cliquez sur B pour lancer l'application » devient :

«1. Appuyer sur la touche A. Une fenêtre s'ouvre.

2. Cliquer sur B. L'application est lancée. »

$51 \quad$ Règle 7

Paralléliser. Tout comme la recherche de synonymes, limiter la recherche de formulations variées "pour éviter la répétition ». Listes et tableaux permettent souvent de contourner ce problème, récurrent en français, langue qui craint la ritournelle.

\section{Exemples}

On 1 September 2009 incandescent bulbs of 100 watts and above will no longer be on sale in

shops in the EU. All 75 watt bulbs will go the following year, and all 60 watt bulbs the year

after that. Bulbs smaller than 60 watts will go on 1 September 2012.

"Dès le $1^{\text {er }}$ septembre 2009, les ampoules incandescentes de 100 watts et plus disparaîtront des magasins de l'UE. Un an plus tard, ce sera au tour de celles de 75 watts, puis 60 watts l'année suivante. Celles de moins de 60 watts ne seront plus vendues à partir du $1^{\text {er }}$ septembre $2012 »$ peut devenir :

Tableau. - Calendrier du retrait des ampoules à incandescence dans l'UE : 


\begin{tabular}{|l|r|l|}
\hline 1. & $\geq 100 \mathrm{~W}$ & $1^{\text {er }}$ sept. 2009 \\
\hline 2. & $75 \mathrm{~W}$ & $1^{\text {er }}$ sept. 2010 \\
\hline 3. & $60 \mathrm{~W}$ & $1^{\text {er }}$ sept. 2011 \\
\hline 4. & $<60 \mathrm{~W}$ & $1^{\text {er }}$ sept. 2012 \\
\hline
\end{tabular}
confort et en toute confiance afin d'atteindre un but (précisons que l'utilisateur peut être un agent de terrain, mais aussi un responsable chargé de gérer un système documentaire). Pour cela, le document doit présenter certaines caractéristiques, dont un contenu expert intégral et non équivoque, mais aussi une rédaction claire, précise et économe, une autorité incontestée et une forme juste. Un contenu expert présenté de manière confuse ou approximative fera perdre du temps à l'utilisateur, tout en lui imposant une charge mentale malvenue, tandis qu'un manque de crédibilité dû à une autorité incertaine ou à un style inadapté fera douter l'utilisateur de l'adéquation du document, l'incitant à le rejeter. La facilité et la confiance avec laquelle l'utilisateur s'approprie et met en œuvre le document expriment la qualité ergonomique de ce dernier. 

documents techniques en les rendant plus simples et plus homogènes, constituent un outil permettant d'améliorer les qualités ergonomiques d'un document destiné à un utilisateur expert, adepte du domaine en question, motivé et contraint par un impératif d'efficacité. D'autre part, les langues contrôlées ont acquis, par leur diffusion dans l'industrie, une valeur normative qui crédibilise les documents techniques qui les utilisent.

Le traducteur technique doit assurer la qualité ergonomique du document cible, même (ou surtout) si celle du document source peut être améliorée, ce qui est très souvent le cas. Cette obligation de service envers l'utilisateur, in fine seul juge de l'acceptabilité du document traduit, doit commander au traducteur de profiter pleinement de la grande marge de manœuvre dont il dispose pour la formulation et l'organisation du document. Les langues contrôlées proposent un ensemble de consignes qui s'intègrent dans les bonnes pratiques de rédaction: le traducteur peut les employer à discrétion afin d'apporter au document technique clarté, précision et concision dans le respect des conventions textuelles du genre.

\section{BIBLIOGRAPHIE}

BARTHE Kathy, « GIFAS Rationalized French: A Controlled Language for Aerospace Documentation in French », Technical Communication, vol. 46, 1999, p. 220-229.

BRINGHURST Robert, The Elements of Typographic Style, Vancouver, Hartley and Marks, 2004, $3^{\mathrm{e}}$ édition, $352 \mathrm{p}$.

Costa Pereira Célia (da) et Pasi Gabriella, « Fuzzy indices of document reliability », Applications of fuzzy sets theory, Heidelberg, Springer-Verlag, 2007, coll. « Lecture Notes in Computer Science, vol. 4578 », p. 110-117.

CORNU Gérard, Linguistique juridique, Paris, Monchrestien, 1990, 412 p.

COUNCIL OF SCIENCE EDITORS, Scientific Style and Format: The CSE Manual for Authors, Editors and Publishers, Council of Science Editors, 2006, $7^{\mathrm{e}}$ édition revisée, $658 \mathrm{p}$.

CRYSTAL David, « Language for special purposes », The Cambridge Encyclopedia of Language, Cambridge, Cambridge University Press, 1997, 2édition, p. 378-393.

FROELIGER Nicolas, « Binaire et liminaire : la forme en traduction technique », Tribune internationale des langues vivantes, $n^{\circ} 45$, novembre 2008 , p. 75-85.

FuchS Norbert E., SCHWERTEL Uta et SCHWITTER Rolf, « Attempto Controlled English - Not Just Another Logic Specification Language », dans P. Flener (éd.), Proceedings of the 8th International Workshop on Logic Programming Synthesis and Transformation, Heidelberg, Springer-Verlag, coll. «Lecture Notes in Computer Science, vol. 1559 », 1999, p. 1-20.

GaVieIRo-Villatte Elisa et Spaggiari Laurent, « Open-ended overview of controlled languages », Bulletin de linguistique appliquée et générale, nº 24, 1999, p. 89-100. 
GouADEC Daniel (éd.), Terminologie et Terminotique : outils, modèles et méthodes, Paris, La Maison du dictionnaire, 1992.

LADMIRAL Jean-René, « Sourciers et ciblistes », Revue d'esthétique, n 12, 1986, p. 33-42.

LAPLACE Colette, Théorie du langage et théorie de la traduction, Paris, Didier Érudition, coll.

«Traductologie, $\mathrm{n}^{\circ} 8$ », 1994, $239 \mathrm{p}$.

MUEGGE Uwe, « Controlled language: the next big thing in translation? », ClientSide News Magazine, Denver CO, ClientSide Publications, vol. 7, nº 7, 2007, p. 21-24.

NAKBI Khédija, «La rédactologie : domaine, méthode et compétences », ASp (revue du GERAS), $\mathrm{n}^{\text {os }}$ 27-38, 2002, p. 15-26.

O'BRIEN Sharon, « Controlling controlled English - an analysis of several controlled language rule sets ", Controlled Translation - Proceedings of the Joint Conference combining the 8th International Workshop of the European Association for Machine Translation and the 4th Controlled Language Application Workshop (EAMT-CLAW03), Dublin City University, Irlande, 2003, p. 105-114.

PeRCeBoIs Jacqueline, « De l'anglais au français en langue de spécialité économique : équivalence attestée et détours des choix traductologiques ", ASp (revue du GERAS), n ${ }^{\text {os }} 45-46,2004$, p. 81-95.

ReIss Katharina, «L'incidence des genres de textes et des conventions textuelles sur l'activité traduisante », dans K. Reiss, Problématiques de la traduction : les conférences de Vienne, Paris, Economica/Anthropos, coll. « Bibliothèque de traductologie », 1995, p. 123-142.

REUTHER Ursula, « Two in one - Can it work? Readability and translatability by means of controlled language ", Proceedings of the Joint Conference combining the 8th International Workshop of the European Association for Machine Translation and the 4th Controlled Language Application Workshop (EAMT-CLAW03), Dublin City University, Irlande, 2003, p. 15-17.

RHEAULT Sylvain, « La Toile fait-elle autorité ? ", Surfaces, Université de Montréal, vol. VIII.105, 1999, p. 4-14.

RYAN Richard, « Les langues contrôlées sont-elles l'avenir de la traduction ? ", La Traductologie de plein champ (volume II), Tribune internationale des langues vivantes, $\mathrm{n}^{\circ} 45,2008$, p. 60-74.

-, «Les langues contrôlées - une valeur ajoutée pour le traducteur », Traduire, n² 220, 2009, p. 57-67.

SWANSON Ellen, Mathematics into Type, Providence RI, American Mathematical Society, 2000, 102 p.

\section{NOTES}

1. Pour une introduction, voir Gouadec (1992).

2. L'identité du rédacteur est néanmoins connue, au moins de l'émetteur, car le document doit être traçable.

3. "Étude scientifique de la relation entre l'homme et ses moyens, méthodes et milieux de travail » (IV Congrès international d'ergonomie, 1969), l'ergonomie s'emploie à développer des systèmes « qui puissent être utilisés avec le maximum de confort, de sécurité et d'efficacité par le plus grand nombre » (Société d'ergonomie de langue française).

4. Utilité : «Aptitude d'un bien à satisfaire un besoin ou à créer les conditions favorables à cette satisfaction » (Petit Larousse illustré, 2008, p. 1051).

5. Commode : « Approprié à l'usage qu'on veut en faire » (Petit Larousse illustré, 2008, p. 226).

6. Praticité : « Caractère de ce qui est pratique, fonctionnel » (Petit Larousse illustré, 2008, p. 812). 
7. Adéquation : «Adaptation parfaite » (Petit Larousse illustré, 2008, p. 15).

8. Justesse : «Qualité d'une chose bien réglée, exacte et donc bien adaptée à sa fonction » (Petit Larousse illustré, 2008, p. 566).

9. Pour une formalisation du concept de fiabilité documentaire, voir da Costa Pereira et Pasi (2007).

10. L'orthotypographie est le premier critère d'authenticité d'un document technique.

11. Par «expert" nous entendons une personne qui produit ou qui utilise un document technique à des fins professionnelles. Un consommateur peut être expert, mais n'a pas vocation à l'être.

12. La première conférence européenne en ergonomie cognitive eut lieu en 1982.

13. Pour un panorama, voir Gavieiro-Villatte et Spaggiari (1999).

14. Un manuel est distribué par ASD-STAN Standardization, avenue de Tervuren, B-1150 Bruxelles, Belgique.

15. Un manuel est disponible en ligne sur le site du Department of Computer Science, Université de Zurich, Suisse.

16. Par exemple, les spécifications ATA iSpec 2200 et S1000D pour la documentation aérospatiale. 17. Le terme "elegant variation" (de l'auteur anglais Henry W. Fowler) ne semble pas avoir d'équivalent satisfaisant en français («synonymie maniérée »?), bien que le calque «variation élégante » soit attesté.

18. Les traductions à modifier portent un astérisque.

19. Pour un traitement détaillé, voir Cornu (1990).

20. Et la concession par le verbe « pouvoir » au présent de l'indicatif, équivalent ici à may.

21. Et non à traits d'union.

22. "Use a tabular layout for complex texts. "

23. "Write for the eye as well as the mind. »

\section{RÉSUMÉS}

L'acceptabilité d'un document technique traduit dépend de son adéquation et de la confiance qu'il inspire à un ensemble d'utilisateurs. Ces deux paramètres dépendent à leur tour des qualités ergonomiques que possède le document. Il appartient au traducteur d'assurer ces qualités ergonomiques en rendant le document facile à s'approprier et à utiliser, et en le dotant d'une architecture apte à conforter sa crédibilité. Les langues contrôlées sont utilisées depuis longtemps dans l'industrie pour la rédaction des documents techniques. Mais elles peuvent aussi aider le traducteur à atteindre les objectifs de la qualité ergonomique.

The acceptability of a translated technical document depends on its usability and the confidence a set of users has in it. Both depend in turn on the ergonomic quality of the document. The translator should secure this ergonomic quality by making the document easy to address and to use, and organizing its form to lend it credibility. Controlled languages have long been used in industry for drafting technical documents. But they can also help the translator to achieve ergonomic quality objectives. 
INDEX

Mots-clés : document technique, ergonomie, langues contrôlées, traduction

Keywords : controlled languages, ergonomics, technical document., translation

\section{AUTEUR}

\section{RICHARD RYAN}

Laboratoire de recherche sur le langage (LRL, EA 999), Université Blaise Pascal (Clermont 2) 